Ophthalmologe 2020 - 117:638-641 https://doi.org/10.1007/s00347-020-01142-1 Online publiziert: 11. Juni 2020

(c) Springer Medizin Verlag GmbH, ein Teil von Springer Nature 2020

Das neue „Severe Acute Respiratory Syndrome Corona-Virus 2" (SARS-CoV-2) hat sich weltweit verbreitet. Seit der ersten Warnung vor einem potenziellen Ausbruch einer neuen Infektionskrankheit durch den Augenarzt Dr. Wenliang Li aus Wuhan, China, hat sich die Welt verändert [13]. Trotz ausführlicher Aufarbeitung vorangegangener Pandemien überschlugen sich die Ereignisse in den ersten Wochen mit zum Teil frappierender Ähnlichkeit zu literarischen und historischen Szenen [3, 8].

SARS-CoV-2 ist als Aerosol und auf Oberflächen stabil [17]. Neben einer gesicherten Übertragungsmöglichkeit durch Tröpfchen, ist auch eine Übertragung über den Tränenfilm denkbar, aktuell aber nicht gesichert [16]. Die größte Infektiosität besteht vor Symptombeginn der durch SARS-CoV-2 ausgelösten „Coronavirus Disease 2019“ (COVID-19) [6]. In Ermangelung einer kausalen Therapie sind daher Schutzmaßnahmen vor Ansteckung und Verbreitung während der COVID-19-Pandemie notwendig.

\section{Medizinberuf als größter Risikofaktor für COVID-19}

Für die Öffentlichkeit existieren Maßnahmen zur Eindämmung der Pandemie wie Mobilitätseinschränkungen mit Reiserestriktionen und Ausgangssperren, Maßnahmen zum Schutz vulnerabler Gruppen und Maßnahmen zur Folgenminderung durch Wahrung sozialer Distanz und Hygieneempfehlungen [15]. Wenn die für die Öffentlichkeit geltenden Maßnahmen in „systemrelevanten Berufen " nicht eingehalten werden können, entstehen erhöhte Risiken und speziel-

Katrin Wacker - Thomas Reinhard

Klinik für Augenheilkunde, Universitätsklinikum Freiburg, Freiburg, Deutschland

\title{
Spezielle ophthalmologische Schutzmaßnahmen in der COVID-19-Pandemie
}

le Anforderungen der Risikoreduktion zum Schutz der Mitarbeiter, denn einer der größten Risikofaktoren für COVID19 ist die Arbeit in Medizinberufen [11]. In Wuhan war die Rate der täglichen Infektion pro 1 Mio. Menschen in Medizinberufen etwa 3-mal so hoch im Vergleich zur Allgemeinbevölkerung [11]. In Italien arbeiteten laut italienischem Gesundheitsministerium über $11 \%$ der COVID-19-positiven Patienten in Medizinberufen [7]. Daher sind besondere Maßnahmen des Arbeitsschutzes in Medizinberufen notwendig.

\section{Herausforderungen und Ziele in der Augenheilkunde}

In der Augenheilkunde sind Arbeitsschutzmaßnahmen von besonderer Bedeutung, da ophthalmologische Untersuchungen in geringem Abstand zum Patienten erfolgen. Maßnahmen zur Risikoreduktion sind jedoch nicht nur zum Schutz für Personal nötig, sondern auch zum Schutz für Patienten, da eine beträchtliche Anzahl ophthalmologischer Patienten altersbedingt zu COVID-Risikogruppen zählen [11]. Ziele spezieller ophthalmologischer Schutzmaßnahmen sind daher einerseits die Aufrechterhaltung der Versorgung, die Vermeidung von Kollateralschäden durch Unterversorgung und andererseits die Vermeidung der Verbreitung von SARS-CoV-2. Angesichts einer unklaren Studienlage zum optimalen Vorgehen variieren Vorgaben und Empfehlungen von Gesetzgebern und Fachgesellschaften zur Risikoreduktion in der Augenheilkunde weltweit (Tab. 1 in [10]).

\section{Überlegungen an der Klinik für Augenheilkunde Freiburg}

Spezielle ophthalmologische Schutzmaßnahmen können in 3 Kategorien eingeteilt werden [10] und werden exemplarisch an Überlegungen und Verfahrensweisen der Klinik für Augenheilkunde Freiburg aufgezeigt (• Abb. 1).

\section{Schutz durch Praxismanagement}

Ein zentrales Element des Krisenmanagements ist die zügige Klärung von Rollen und Aufgaben [5]. Insbesondere in Extremsituationen wird deutlich, wie wichtig funktionierende Kommunikations- und Rapportstrukturen sind, um Mitarbeiter transparent und effektiv zu führen. Innerhalb der Augenklinik wurde ein Krisenstab mit zentralen Entscheidungsträgern etabliert, der der Klinikumseinsatzleitung des gesamten Universitätsklinikums Freiburg nachgeschaltet war. Durch regelmäßige Treffen konnten schnell Konzepte etabliert, angepasst und den Mitarbeitern, Patienten und Zuweisern kommuniziert werden. Im Detail wurde die Notfallversorgung durch folgende Maßnahmen aufrechterhalten:

- Der Zugang zur Klinik wurde auf Mitarbeiter mit Ausweis und auf $\mathrm{Pa}$ tienten beschränkt. Begleitpersonen

\begin{tabular}{|ll}
\hline \multicolumn{2}{|l}{ Abkürzungen } \\
\hline COVID-19 & Coronavirus Disease 2019 \\
\hline FFP & Filtering face piece \\
\hline SARS-CoV-2 & $\begin{array}{l}\text { Severe Acute Respiratory } \\
\text { Syndrome Corona Virus 2 }\end{array}$ \\
\hline
\end{tabular}


Identifikation von COVID-19-Verdachtsfällen

\section{Wachdienst - Zugangsbeschränkung}

- Patient erhält Mund-Nasen-Schutz, Händedesinfektion

2. Triage am Eingang

JA
Triage Ambulanz/Operationszentrum NEIN
NEIN

Bei jedem Patienten alle 5 Fragen vollständig stellen, keine Interpretation:

1. Wurden Sie positiv auf Corona getestet oder hatten Kontakt zu einer infizierten Person?

2. Haben Sie Husten, Schnupfen oder Schwierigkeiten zu atmen?

3. Haben Sie Fieber oder Schüttelfrost?

4. Haben Sie Hals-, Kopf- oder Gliederschmerzen?

5. Ist Ihr Geruchs- oder Geschmackssinn verändert ?

\begin{tabular}{|c|c|}
\hline ISOLATIONSBEREICH & Ambulanz/Station/OP \\
\hline \multicolumn{2}{|c|}{ 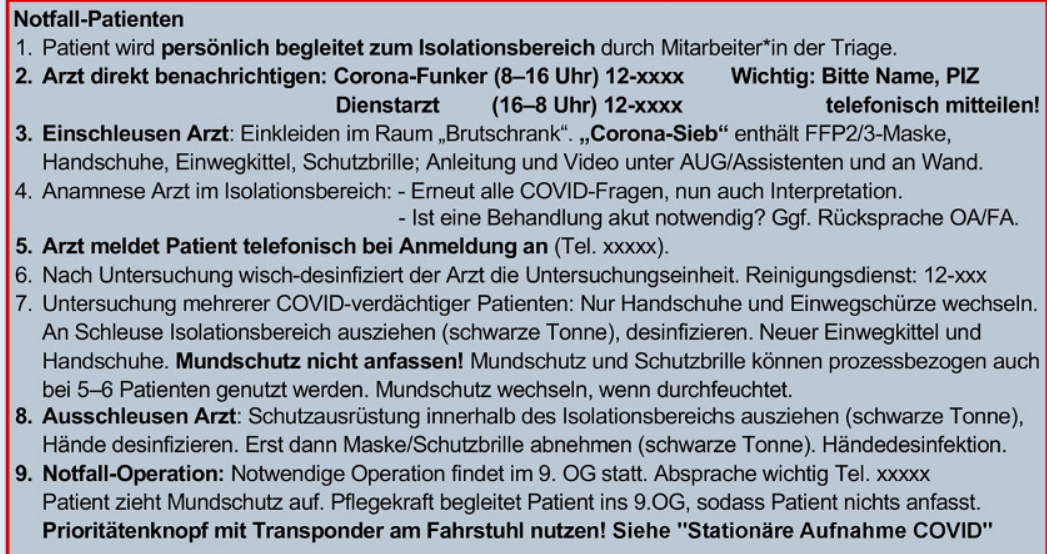 } \\
\hline $\begin{array}{l}\text { mit Namen, PIZ und Information; neuer Termin frühestens } 2 \text { Wochen } \\
\text { 3. Stationäre Operation } \rightarrow \text { Patient in Isolationsbereich: Corona-Fu } \\
\text { Dienstarzt }\end{array}$ & $\begin{array}{l}\text { ch Hause. E-Mail an AUG-IVOM } \\
\text { Beschwerdefreiheit. } \\
\text { Hause. E-Mail an AUG-TV } \\
\text { ch Beschwerdefreiheit. } \\
\text { (8-16Uhr) 12-xxxxx } \\
\text { (16-8 Uhr) 12-xxxx, RS OAFA }\end{array}$ \\
\hline r Medizin Tel. ,,xxxxxx“ & ggf. Monitorpflichtigkeit: \\
\hline
\end{tabular}

Abb. 1 ॥ Flussdiagramm COVID-19-Triage der Klinik für Augenheilkunde Freiburg. (๑ Die Autoren, mit freundl. Genehmigung)

erhielten nur in Ausnahmen $\mathrm{Zu}$ gang, z. B. bei Kindern, gesetzlicher Betreuung oder Patienten aus der Justizvollzugsanstalt.

- Die Sektionsleiter führten eine inhaltliche Triage aller geplanten Ambulanz- und Operationstermine durch, um Notfälle und dringliche Kontrollen zu identifizieren. Die Absage von elektiven und nichtdringlichen Fällen erfolgte initial fernmündlich, im Verlauf postalisch. Beispiele einer möglichen Triage sind in EyeWiki oder im Krisenplan bettenführender Kliniken aufgelistet $[4,9]$.

- Es wurden Telemedizinlösungen für postoperative Kontrollen z. B. nach intravitrealen Injektionen angeboten. Hierfür erhielten Patienten, deren niedergelassener Augenarzt keine Kontrollen anbieten konnte, einen Gutschein für eine Video- und Telefonsprechstunde.

- Die Identifikation von Patienten mit COVID-Verdacht erfolgte an 3 Stellen: vor dem Termin durch
Kontaktaufnahme mit einem Telefonleitfaden, am Eingang der Klinik und erneut vor der ersten Untersuchung in der Hochschulambulanz (• Abb. 1).

- Die Koordination des Patientenflusses im Haus erfolgte durch erfahrene Pflegekräfte der Hochschulambulanz und durch Mitarbeiter der Anmeldung, die als Patientenscouts bereits vor der COVID-Pandemie tätig waren.

- Die Aufenthaltsdauer von Patienten wurde durch Reduktion der Termine pro Zeiteinheit und durch eine Verringerung der Funktionsdiagnostik erreicht.

- IT-Lösungen zur Patientenleitung wurden erarbeitet und verbessert. Zum Beispiel wurde die elektronische Planung der Visite mit Terminierung notwendiger Funktionsdiagnostik weiterentwickelt.

- Die Kommunikation der aktuellen Regeln erfolgte über die Webseite der Klinik und postalisch an Patienten und niedergelassene Augenärzte des Augennetzes Südbaden.

- Die Mitarbeiter des Patiententelefons erhielten eine Schulung zum Thema „Häufige Fragen zu Corona und einheitliche Antworten“.

\section{Schutz der Mitarbeiter}

$\mathrm{Zu}$ zentralen Elementen des Mitarbeiterschutzes gehören Maßnahmen über die Umsetzung der in der Öffentlichkeit geltenden Schutzmaßnahmen hinaus. Die Deutsche Gesetzliche Unfallversicherung (DGUV 112-190) sieht beispielsweise in normalen Arbeitsplatzsituationen für den Umgang mit Viren einen Atemschutz mittels FFP3-Masken vor (engl. „filtering face piece" [FFP]). Die Einteilung der FFP-Masken erfolgt entsprechend dem prozentualen Anteil der zu filternden Partikel (80-99\%) und der Gesamtleckage (maximal 25-5\%). Unabhängig vom COVID-Status wird daher bei Patientenkontakt, der zu Husten- oder Niesreiz führen kann, das Tragen eines Atemschutzes am Universitätsklinikum Freiburg empfohlen. In der Augenklinik tragen alle Mitarbeiter bei der Fundoskopie und der Spalt- 
lampenuntersuchung einen Atemschutz. Wertvolle Ressourcen und Übersichten zum Mitarbeiterschutz bieten die Webseiten des Robert Koch-Instituts, der American Academy of Ophthalmology und das "Séparée“ des Berufsverbands der Augenärzte Deutschlands e. V. [1, 2, 14].

- Es erfolgten Anweisungen zur Einhaltung erprobter und evidenzbasierter Standardhygienemaßnahmen wie Hände- und Wischdesinfektion. Alle Mitarbeiter wurden angehalten, die Bereichskleidung des Klinikums zu verwenden.

- Der korrekte Umgang mit persönlicher Schutzausrüstung wie Schutzkleidung oder Schutzbrille bei COVID-verdächtigen Patienten wurde den Mitarbeitern mit Bildstrecken und Videos erklärt.

- Alle Patienten erhielten vor der Eingangstür durch den Sicherheitsdienst einen sauberen Mund-Nasen-Schutz, da die Tauglichkeit eigenständig hergestellter Masken zum Selbst- und Fremdschutz variiert [12]. Alle Mitarbeiter wurden angehalten, einen Mund-Nasen-Schutz zu tragen.

- In den ersten Wochen der COVID19-Pandemie erfolgte eine Reduktion des Personals vor Ort in wochenweisem Turnus, um die Infektions- und Übertragungsmöglichkeiten zu verringern und personelle Reserven zur Aufrechterhaltung der Versorgung zu bilden. Ein Team vor Ort sorgte für die Aufrechterhaltung der reduzierten Patientenversorgung. Das andere Team entwickelte im „Home-Office“ wissenschaftliche Arbeiten, Prozessdokumente und digitale Lehrinhalte der studentischen Lehre.

- Alle Fort- und Weiterbildungsveranstaltungen mit internen und externen Referenten wurden abgesagt.

- Sollte zukünftig ein Mitarbeiter positiv auf SARS-CoV-2 getestet oder das positive Testergebnis eines Patienten nachträglich mitgeteilt werden, kann ein definierter Maßnahmenkatalog zum Umgang mit Kontaktpersonen und notwendiger Desinfektion im Zweifelsfall ein schnelles Handeln ermöglichen.

Ophthalmologe 2020 · 117:638-641 https://doi.org/10.1007/s00347-020-01142-1

๑) Springer Medizin Verlag GmbH, ein Teil von Springer Nature 2020

\section{K. Wacker · T. Reinhard}

\section{Spezielle ophthalmologische Schutzmaßnahmen in der COVID-19-Pandemie}

\section{Zusammenfassung}

Hintergrund. Die Arbeit in einem Medizinberuf ist ein Risikofaktor für Coronavirus Disease 2019 (COVID-19).

Fragestellung. Der Artikel fasst spezielle Schutzmaßnahmen im Rahmen der COVID19-Pandemie in der Augenheilkunde zusammen.

Material und Methoden. Spezielle Schutzmaßnahmen zur Aufrechterhaltung der ophthalmologischen Versorgung, Vermeidung von Kollateralschäden durch Unterversorgung und des Infektionsschutzes vor dem "Severe Acute Respiratory Syndrome Corona-Virus 2" (SARS-CoV-2) werden am Beispiel eines tertiären Referenzzentrums dargestellt.

Ergebnisse. Der Schutz durch Praxismanagement reichte von Zugangsbeschränkungen und Triageprotokollen über Strategien zur kürzeren Aufenthaltsdauer bis hin zu Telemedizinlösungen und wurde von einer transparenten Informationspolitik begleitet.
Der Schutz der Mitarbeiter und Patienten basierte auf Standardhygienemaßnahmen wie Hände- und Wischdesinfektion. Um Arbeitsschutz vor Aerosolen zu gewährleisten, war die (Nach-)Schulung und die Verwendung persönlicher Schutzausrüstung notwendig. Bauliche Maßnahmen trugen dazu bei, Mitarbeiter und Patienten bei ophthalmologischen Untersuchungen zu schützen.

Diskussion. Um die ophthalmologische Versorgung während der COVID-19Pandemie aufrechtzuerhalten, wurden Schutzmaßnahmen für Mitarbeiter und Patienten verwendet, die teils über die in der Öffentlichkeit geltenden Bestimmungen hinausgingen.

Schlüsselwörter

Praxismanagement · Ambulante Versorgung . Telemedizin · Notfallversorgung

\section{COVID-19 preparedness in ophthalmology}

\section{Abstract}

Background. Healthcare work is a risk factor for coronavirus disease 2019 (COVID-19). Objectives. To review risk mitigation strategies in ophthalmology during the COVID-19 pandemic.

Material and methods. Risk mitigation strategies to maintain ophthalmology care, to prevent collateral damage from care disruption, and to prevent the spread of severe acute respiratory syndrome coronavirus 2 (SARS-CoV-2) are summarized based on the example of a tertiary referral center in Germany.

Results. Clinical management strategies included restricted access to buildings, triage systems, telemedicine approaches, strategies for rapid treat and release, and transparent communication strategies. Strategies to protect patients and staff relied on both standard hygiene precautions and the use of personal protective equipment for prevention of droplet infections. Physical barriers supported risk mitigation strategies. Conclusion. To maintain ophthalmological care during the COVID-19 pandemic, a coordinated, multifaceted approach using risk mitigation strategies to protect staff, patients, and the public was initiated.

Keywords

Practice management - Outpatient clinic . Telemedicine - Continuity of operations plan

\section{Schutz durch bauliche Maßnahmen}

Das veränderte Praxismanagement erforderte bauliche Maßnahmen, die schrittweise auf eine Pandemie unklarer Dauer angepasst wurden.

- Chronologisch vom Eingangsbereich bis hin zur Untersuchung konnte die
Einhaltung des zwischenmenschlichen Abstandhaltens durch bauliche Veränderungen unterstützt werden, z. B. durch Reduktion der Anzahl der Sitzgelegenheiten oder durch Zuweisung eines Sitzplatzes.

- Sollte die Versorgung COVID-19verdächtiger oder positiv getesteter Patienten mit ophthalmologischen 
Notfällen an Kliniken der Maximalversorgung nötig sein, sind getrennte Warteräume oder ein Isolationsbereich möglich. In Freiburg wurde ein Isolationsbereich installiert mit sterilisierbaren 20-D-Lupen und Einmalinstrumenten wie EinmalTonometer-Messkörper.

- Trennvorrichtungen zum Schutz vor größeren, potenziell infektiösen Partikeln bei Funktionsuntersuchungen, an der Spaltlampe und an Tresen, bekannt als „Spuckschutz“, sind auch in vielen Bereichen außerhalb der Augenheilkunde zu finden.

\section{Ausblick in den Alltag einer Pandemie}

Die nächsten Schritte bestehen darin, aus den Akutmaßnahmen Strukturen zu entwickeln, die auch in den nächsten Monaten Bestand haben und einen Alltag in der ophthalmologischen Versorgung erlauben. Beispielsweise werden Telemedizinlösungen zur Einschätzung und ersten Beratung bei bestimmten Patientengruppen weiterentwickelt und provisorische Hinweisschilder für Verhaltensregeln gegen solidere und maßgeschneiderte Lösungen ausgetauscht.

Die Umsetzung etablierter Pandemiepläne und spezieller Maßnahmen liegt maßgeblich in der Verantwortung aller Mitarbeiter und aller Patienten. Um Verantwortung für Mitarbeiter, Patienten und die Gesellschaft zu übernehmen, sind eine stetige Evaluation und situationsangepasste Reevaluation der Maßnahmen und deren Lockerung unabdingbar und können helfen, das fragile Gleichgewicht zwischen notwendiger medizinischer Versorgung, Arbeitsschutz und Infektionsschutz auszutarieren.

\section{Fazit für die Praxis}

- Ziele spezieller ophthalmologischer Schutzmaßnahmen sind einerseits die Aufrechterhaltung der Versorgung, die Vermeidung von Kollateralschäden durch Unterversorgung und anderseits die Vermeidung der Verbreitung von SARS-CoV-2.
- Um die ophthalmologische Versorgung während der COVID-19Pandemie aufrechtzuerhalten, müssen Mitarbeiter und Patienten über die in der Öffentlichkeit geltenden Maßnahmen hinaus geschützt werden.

- Schutz durch Praxismanagement reicht von Zugangsbeschränkungen über Strategien zur kürzeren Aufenthaltsdauer zu einer transparenten Informationspolitik.

- Der Schutz der Mitarbeiter und Patienten basiert auf erprobten Standardhygienemaßnahmen wie Händeund Wischdesinfektion. Um Arbeitsschutz vor Aerosolen zu gewährleisten, sind die (Nach-)Schulung und die Verwendung persönlicher Schutzausrüstung notwendig.

- Bauliche Maßnahmen unterstützen das Praxismanagement und erlauben einen Schutz der Mitarbeiter und Patienten bei speziellen ophthalmologischen Untersuchungen.

\section{Korrespondenzadresse}

\section{Dr. med. Katrin Wacker}

Klinik für Augenheilkunde, Universitätsklinikum Freiburg

Killianstr. 5, 79106 Freiburg, Deutschland katrin.wacker@uniklinik-freiburg.de

Förderung. Berta-Ottenstein-Programme für Clinican Scientists, Medizinische Fakultät, Universität Freiburg (K. W.)

\section{Einhaltung ethischer Richtlinien}

Interessenkonflikt. K. Wacker und T. Reinhard geben an, dass kein Interessenkonflikt besteht. K. Wacker ist Consultant für ProQR Therapeutics für Projekte unabhängig von diesem Artikel.

Für diesen Beitrag wurden von den Autoren keine Studien an Menschen oder Tieren durchgeführt. Für die aufgeführten Studien gelten die jeweils dort angegebenen ethischen Richtlinien.

\section{Literatur}

1. American Academy of Ophthalmology (2020) Important coronavirus updates for ophthalmologists. https://www.aao.org/headline/alert-importantcoronavirus-context. Zugegriffen: 5. Jan. 2020

2. Berufsverband der Augenärzte Deutschlands e.V. https://www.augeninfo.de/mitglieder.php. Zugegriffen: 1.Mai 2020
3. Camus A (1947) La Peste. Librairie Éditions Gallimard, Paris

4. Deutsche Ophthalmologische Chefärzte (DOCH), Retinologische Gesellschaft (RG), Vereinigung Ophthalmologischer Lehrstuhlinhaber (VOL), Deutsche Ophthalmologische Gesellschaft (DOG). Krisenplan Ophthalmologische nicht-elektive Basisversorgung in bettenführenden Kliniken. https://www.dog.org/wp-content/uploads/ 2020/03/Ophthalmologische-Basisversorgung_ Krisenplan-DOCH_VOL_DOG_RG_31-03-20_ Update-2.pdf.Zugegriffen:20. Mai 2020

5. Federal Emergency Management Agency. Pandemic influenza-Continuity of Operations Plan (COOP). https://www.aao.org/Assets/ 213f04b4-1e30-40a4-9f37-9abe6f76e81c/ 637203302867000000/ophthalmology-pi-coop3 -stripped-rev3-pdf?inline $=1$. Zugegriffen: 1 . Mai 2020

6. HeX, Lau EHY, Wu Pet al (2020) Temporal dynamics in viral shedding and transmissibility of COVID-19. Nat Med. https://doi.org/10.1038/s41591-0200869-5

7. Istituto Superiore Di Sanità (2020) Epidemia COVID-19. https://www.epicentro.iss.it/coronavirus/ bollettino/Bollettino-sorveglianza-integrataCOVID-19_23-aprile-2020.pdf. Zugegriffen: 23. Apr. 2020

8. Morens DM, Taubenberger JK (2018) Influenza cataclysm, 1918. NEngl J Med 379:2285-2287

9. Nguyen MT, Jung H, Taylor LE et al. Coronavirus (COVID-19). https://eyewiki.aao.org/ Coronavirus_(COVID-19).Zugegriffen:1.Mai 2020

10. Li OJP, Shantha J, Wong TY et al (2020) Preparedness among ophthalmologists: during and beyond the COVID-19 pandemic. Ophthalmology 127:569-572

11. Pan A, Liu L, Wang C et al (2020) Association of public health interventions with the epidemiology of the COVID-19 outbreak in Wuhan, China. JAMA. https://doi.org/10.1001/jama.2020.6130

12. Parker-Pope T (2020) What's the best material for a mask? https://www.nytimes.com/ article/coronavirus-homemade-mask-materialDIY-face-mask-ppe.html. Zugegriffen: 28. Apr. 2020

13. Parrish RK 2nd, Stewart MW, Duncan Powers SL (2020) Ophthalmologists are more than eye doctors-in memoriam Li Wenliang. Am J Ophthalmol. https://doi.org/10.1016/j.ajo.2020.02.014

14. Robert-Koch-Institut. COVID-19 (Coronavirus SARS-CoV-2). https://www.rki.de/DE/Content/ InfAZ/N/Neuartiges_Coronavirus/nCoV.html. Zugegriffen: 1. Mai 2020

15. Robert-Koch-Institut. Nationaler Pandemieplan 2017 Teil I. https://www.gmkonline.de/ documents/pandemieplan_teil-i_1510042222 1585228735.pdf.Zugegriffen: 1. Mai 2020

16. Seitzman GD, Doan T (2020) No time for tears. Ophthalmology. https://doi.org/10.1016/j. ophtha.2020.03.030

17. Van Doremalen N, Bushmaker T, Morris DH et al (2020) Aerosol and surface stability of SARSCoV-2 as compared with SARS-coV-1. N Engl J Med 382:1564-1567 